\title{
Efficacy of parasitological methods for the diagnosis of Strongyloides stercoralis and hookworm in faecal specimens
}

\author{
Elizabete de J. Inês a , Joelma N. Souza ${ }^{a}$, Renata C. Santos ${ }^{a}$, Eliane S. Souza ${ }^{a}$, Fred L. Santos ${ }^{a}$, \\ Mônica L.S. Silva ${ }^{a}$, Moacir P. Silva ${ }^{b}$, Márcia C.A. Teixeira ${ }^{a}$, Neci M. Soares ${ }^{a}, *$ \\ a Departamento de Análises Clínicas e Toxicológicas, Faculdade de Farmácia, Universidade Federal da Bahia, Salvador, Brazil \\ b Instituto de Ciências da Saúde, Universidade Federal da Bahia, Salvador, Bahia, Brazil
}

\section{A R T I C L E I N F O}

\section{Article history:}

Received 21 March 2011

Received in revised form 18 August 2011

Accepted 19 August 2011

Available online 27 August 2011

\section{Keywords:}

Strongyloides stercoralis

Hookworm

Agar plate culture

Diagnosis

\begin{abstract}
A B S T R A C T
To compare the efficacy of stool examination for the detection of Strongyloides stercoralis and hookworm, a total of 634 stool samples from the routine laboratory service of the Pharmacia Faculty, Federal University of Bahia, Brazil, were examined by agar plate culture (APC), Baermann-Moraes and spontaneous sedimentation. The sensitivity of agar plate culture, calculated by combining results of all 3 methods, was 95\% for S. stercoralis and 77.6\% for hookwoorm. Moreover, APC had superior accuracy than BaermannMoraes and spontaneous sedimentation for $S$. stercoralis and hookworm diagnosis, respectively. The $S$. stercoralis and hookworm positive samples from the laboratory routine, obtained after the previous analysis, along with those initially selected, were used to evaluate the concordance between microscopic examination and both the type of furrows left by larvae and the time for culture positivity using the APC method. Of 115 stool samples positive for S. stercoralis and 92 positive for hookworm, 110 (95.7\%) and 89 (96.7\%), respectively, had concordant results for furrows and morphological characteristics. The cumulative percentage of positivity increased to $94 \%$ by the third day of observation; at this time, only $19.6 \%$ of hookworm-positive samples had positive culture plates. Analyses of $74 \mathrm{~S}$. stercoralis-positive stool samples stored at $4{ }^{\circ} \mathrm{C}$ for 24,48 and $72 \mathrm{~h}$ showed the presence of larvae in $48.6 \%, 28.4 \%$ and $23 \%$ of samples, respectively when re-examined by the APC. As a definitive diagnosis of strongyloidiasis depends on the microscopic demonstration of parasites, increasing the sensitivity of the detection requires the use of different parasitological methods, including APC.
\end{abstract}

(ㄷ) 2011 Elsevier B.V. All rights reserved.

\section{Introduction}

Strongyloides stercoralis is a soil-transmitted intestinal nematode widely distributed in tropical and sub-tropical regions. It is estimated that 30-100 million individuals worldwide are infected (Ardiç, 2009; Olsen et al., 2009), with higher prevalence in areas with poor hygiene and sanitation (Borda et al., 1996; Menezes et al., 2008; Gamboa et al., 2009). The parasitological diagnosis of $S$. stercoralis infection usually relies on the detection of larvae in stool samples. However, the majority of cases involve a chronic infection, with an intermittent and small larvae load in the faeces, decreasing the sensitivity of parasitological tests (Dreyer et al., 1996; Uparanukraw et al., 1999). Several coprological methods have been used to detect larvae in stool samples, including direct faecal smears, the Baermann-Moraes method, spontaneous sedi-

* Corresponding author at: Rua Barão de Jeremoabo, s/n Campus Universitário de Ondina, Ondina, 40170 115, Salvador, Bahia, Brazil. Tel.: +55 7132836950 ; fax: +557132836919 .

E-mail addresses: necisoares@gmail.com,neci@ufba.br(N.M. Soares). mentation, formalin-ether concentration, filter paper culture and agar plate culture (Arakaki et al., 1990; Kobayashi et al., 1996; Siddiqui and Berk, 2001). Recently, the detection of parasite DNA in faecal samples using real-time PCR proved to be a sensitive and specific method for the diagnosis of S. stercoralis (Verweij et al., 2009; Kramme et al., 2011), although it is not readily adaptable for use in the clinical settings where laboratory facilities are often limited.

Studies have demonstrated the high efficiency of the agar plate culture (APC) method, which can detect more than $90 \%$ of positive cases of strongyloidiasis, even when only a few worms are present (Arakaki et al., 1990; Koga et al., 1992; Dreyer et al., 1996; Hirata et al., 2007). Moreover, for parasitological methods dependent on the movement/migration of live rhabditoid larvae, the use of fresh stool samples that have not been refrigerated is recommended, although no systematic studies have been performed to support this recommendation.

Some authors proposed the analysis of the furrows left by $S$. stercoralis larvae and hookworms on the surface of agar to assist in the diagnosis. The furrows left by $S$. stercoralis larvae are relatively regular, with a sine-curve-like pattern, and are usually in a rather straight or gently curved direction, whereas the furrows 
left by hookworms have an irregular twisting pattern, with abrupt changes in direction (Koga et al., 1990; Dos Santos Neto, 1993; Salazar et al., 1995). Jongwutiwes et al. (1999) have exploited the characteristics of furrows left on agar surfaces by $S$. stercoralis larvae or adult worms and by hookworm larvae, suggesting that the characteristic furrow patterns left by both species can be used in preliminary identification.

In the present study, we confirmed the efficacy of APC, compared to other parasitological methods, as well as, the potential of larval trials morphology as a complementary tool for identification of S. stercoralis and hookworm. Furthermore, to our knowledge, this study has shown the real effect of stool refrigeration on $S$. stercoralis diagnosis for the first time.

\section{Materials and methods}

\subsection{Samples details}

The study was carried out from January 2007 to April 2008 in individuals attending the Clinical Analysis Laboratory of Pharmacia College, Federal University of Bahia, Brazil, where approximately 100 stool samples per day are examined for enteroparasites. First, to compare parasitological diagnostic methods for S. stercoralis and hookworm 6-8 samples were randomly selected daily, up to a total of 634 faeces. All of these faecal samples were examined by 3 different methods: spontaneous sedimentation, the Baermann-Moraes and the APC. Second, the S. stercoralis and hookworm positive samples from the laboratory routine, obtained after the previous analysis, along with those initially selected, were used to evaluate the agreement between microscopic examination and both the type of furrows left by larvae and the time for culture positivity using the APC method, as described below. This study was approved by the Committee of Ethics in Research of the Gonçalo Moniz Institute, Oswaldo Cruz Foundation, and an informed consent for participation was obtained from each patient during delivery of clinical specimens.

\subsection{Stool examination by APC}

To compare the efficacy of APC with that of conventional methods and to analyze the characteristics of the mobility patterns of S. stercoralis and hookworm larvae on agar plates, a total of 210 positive samples identified by Baermann-Moraes and spontaneous sedimentation methods ( 115 for S. stercoralis, 92 for hookworm and 3 for both parasites) were used within $2 \mathrm{~h}$ after delivery to the laboratory. Agar plate cultures were performed by placing $3 \mathrm{~g}$ of faeces on sterile plastic Petri dishes (diameter $8.5 \mathrm{~cm}$ and depth $1.5 \mathrm{~cm}$ ) containing approximately $5 \mathrm{~mL}$ of nutrient agar (1.5\% agar, $0.5 \%$ beef extract, $1.0 \%$ peptone and $0.5 \%$ sodium chloride). Dishes were sealed with adhesive tape to prevent larvae from crawling out of the plate and were incubated at $32^{\circ} \mathrm{C}$ (Koga et al., 1991). As the larvae crawled over the agar, they carried bacteria with them, creating visible tracks. Plates were checked daily for the presence of crawling marks, moving larvae or free-living adults (S. stercoralis samples) for up to 7 days, as described by Koga et al. (1991). After the crawling marks were traced, larvae were recovered by washing the agar surface with phosphate-buffered formalin and were identified by considering the following morphological characteristics. Larvae with bulbed esophagus (rhabditiform stage) with short buccal cavity (length about one third of the width of the larvae anterior end), visible genital primordium and the tail ending abruptly were identified as S. stercoralis, while rhabditiform larvae with long buccal cavity (as long as the width of the body), inconspicuous genital primordium and an end of tail elongated were identified as hookworm. Filariform stages (lacking prominent bulb in esophagus and size range from 500 to $700 \mu \mathrm{m}$ long by $20-24 \mu \mathrm{m}$ of width) with esophagus approximately $1 / 2$ length of body and a notched tail were identified as $S$. stercoralis while larvae with shorter esophagus (1/4 length of body) and pointed tail were diagnosed as hookworm.

Plates were examined microscopically under $100 \times$ magnification. The assessment of the characteristics of the furrows was made independently by 3 observers without knowledge of the results of the microscopic examination. The result was considered concordant only if all 3 observers had identified the same pattern of larvae furrows and if this pattern was compatible to larvae morphology.

\subsection{Evaluation of the influence of stool refrigeration on $S$. stercoralis larvae recovery}

Seventy-four fresh stool samples from patients with S. stercoralis infection confirmed by the Baermann-Moraes method were extensively homogenised. Approximately $3 \mathrm{~g}$ were used to perform the APC, and the remainder of the faecal material was stored at $4{ }^{\circ} \mathrm{C}$. After 24,48 and $72 \mathrm{~h}$ of storage, the samples were re-examined to evaluate the presence of $S$. stercoralis by the Baermann-Moraes and APC methods.

\subsection{Statistical analysis}

The data were processed using the SPSS program 15.0 for Windows. Comparison of APC with Baermann-Moraes and spontaneous sedimentation methods and differences in time for APC positivity for S. stercoralis and hookworm diagnosis were performed by Fisher's exact test. All of the statistical analyses were performed using the statistical software GraphPad (San Diego, USA). A P value $\leq 0.05$ was considered significant.

\section{Results}

The overall frequencies of $6.6 \%$ for S. stercoralis and $7.7 \%$ for hookworm were obtained, when considering the combination of results from the 3 different parasitological methods. The APC method was the most sensitive for the detection of both S. stercoralis (95\%) and hookworm (77.6\%). The APC increased the number of $S$. stercoralis positive samples detected by Baermann-Moraes and spontaneous sedimentation methods by 1.38 and 3.64 times, diagnosing an additional of 11 and 29 cases, respectively (Table 1 ). Spontaneous sedimentation had the lowest sensitivity for the recovery of S. stercoralis (27.5\%), identifying rhabditoid larvae in 11 of the 40 positive stool samples (Table 1 ). As expected, spontaneous sedimentation had more sensitivity in detecting hookworm (73.7\%) than Baermann-Moraes (21\%), since eggs are the common parasite stage excreted in faeces (Table 1). The APC method also detected 10 additional cases of hookworm that were not diagnosed by the conventional method of spontaneous sedimentation (Table 1). Pair-wise comparisons of APC with Baermann-Moraes and spontaneous sedimentation to detect $S$. stercoralis and hookworm larvae revealed statistically significant difference $(P=0.0001)$.

As previously described by other authors, the analysis of agar plates confirmed the different mobility patterns of S. stercoralis and hookworm larvae (Fig. 1). The results for the characteristics of furrows left by the larvae and morphological identification were 95.7\% (110/115), 96.7\% (89/92) and 100\% (3/3) concordant for faecal samples positive for $S$. stercoralis, hookworm and both parasites, respectively (Table 2 ). In less than $10 \%$ of S. stercoralis cultures, despite the presence of crawling marks, no larvae was recovered after washing, or inversely, recuperation of larvae without visible tracks. These samples were pulled out from the study of concordance between trails pattern and larvae morphology. 
Table 1

Comparison of agar plate culture (APC) with Baermann-Moraes and spontaneous sedimentation methods for S. stercoralis and hookworm diagnosis.

\begin{tabular}{|c|c|c|c|c|c|c|c|c|c|}
\hline & & \multicolumn{8}{|c|}{ Agar plate culture ${ }^{\mathrm{a}}$} \\
\hline & & \multicolumn{4}{|c|}{ Strongyloides stercoralis } & \multicolumn{4}{|c|}{ Hookworm } \\
\hline & & Positive & Negative & Total & Sensitivity (\%) & Positive & Negative & Total & Sensitivity (\%) \\
\hline \multirow[t]{3}{*}{ Baermann-Moraes } & Positive & 29 & 2 & 31 & \multirow[t]{3}{*}{72.5} & 8 & 0 & 8 & \multirow[t]{3}{*}{21} \\
\hline & Negative & 11 & 592 & 603 & & 30 & 596 & 626 & \\
\hline & Total & 40 & 594 & 634 & & 38 & 596 & 634 & \\
\hline \multirow{3}{*}{$\begin{array}{l}\text { Spontaneous } \\
\text { sedimentation }\end{array}$} & Positive & 11 & 0 & 11 & \multirow[t]{3}{*}{27.5} & 28 & 11 & 39 & \multirow[t]{3}{*}{73.7} \\
\hline & Negative & 29 & 594 & 623 & & 10 & 585 & 595 & \\
\hline & Total & 40 & 594 & 634 & & 38 & 596 & 634 & \\
\hline
\end{tabular}

a Comparison of APC with Baermann-Moraes and spontaneous sedimentation to detect $S$. stercoralis and hookworm larvae was statistically significant $(P=0.0001)$. The sensitivity of agar plate culture, calculated by combining results of all 3 methods, was 95\% (40/42) for S. stercoralis and 77.6\% (38/49) for hookwoorm.
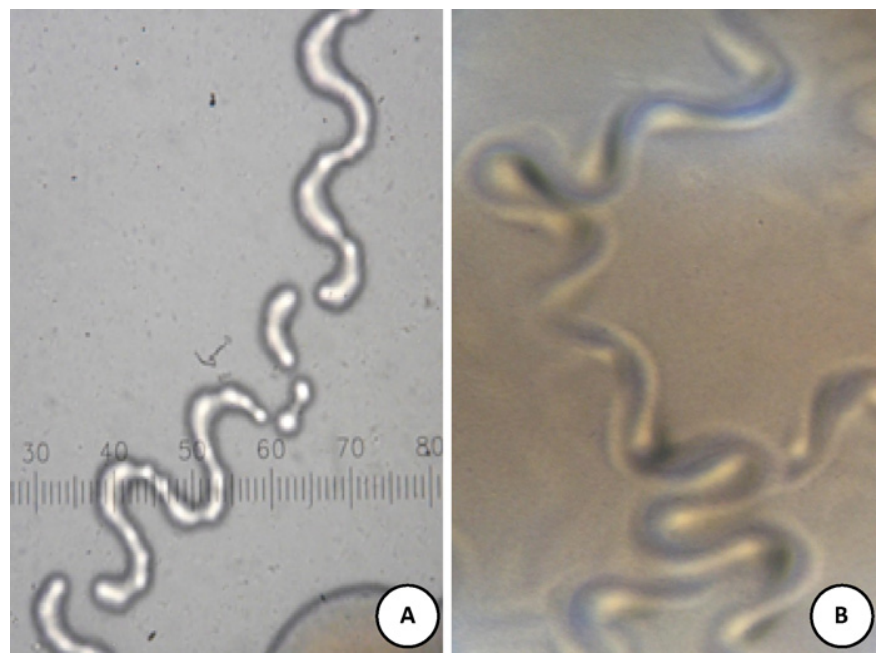

Fig. 1. Characteristic tracks or furrows left by the crawling of $S$. stercoralis (A) and the hookworm (B) larvae on the agar plate under microscope (100× magnification), as described in Section 2.

The diagnosis of $S$. stercoralis and hookworm using APC also varied according the incubation time of the plates. Of the $115 \mathrm{~S}$. stercoralis-positive faecal samples, 73 (63.5\%) were detected by APC in the first day (Fig. 2). The cumulative percentage of positivity increased to $94 \%$ by the third day of observation. In contrast, only 3 stool samples (3.3\%) were positive for hookworm on the first day of culture $(P=0.0001)$, increasing to $90 \%$ detection on the fifth day of incubation (Fig. 2). Three stool samples positive for both parasites were positive in the third day of incubation (data not shown).

The storage of faeces in a refrigerator interfered with the parasitological diagnosis of strongyloidiasis. Analyses of 74 positive stool samples stored at $4{ }^{\circ} \mathrm{C}$ for 24,48 and $72 \mathrm{~h}$ showed the presence of larvae in 36 (48.6\%), 21 (28.4\%) and 17 (23\%) of samples, respectively when re-examined by APC (Table 3 ). Similar results were also observed when samples were re-examined by the BaermannMoraes method, as shown in Table 3.

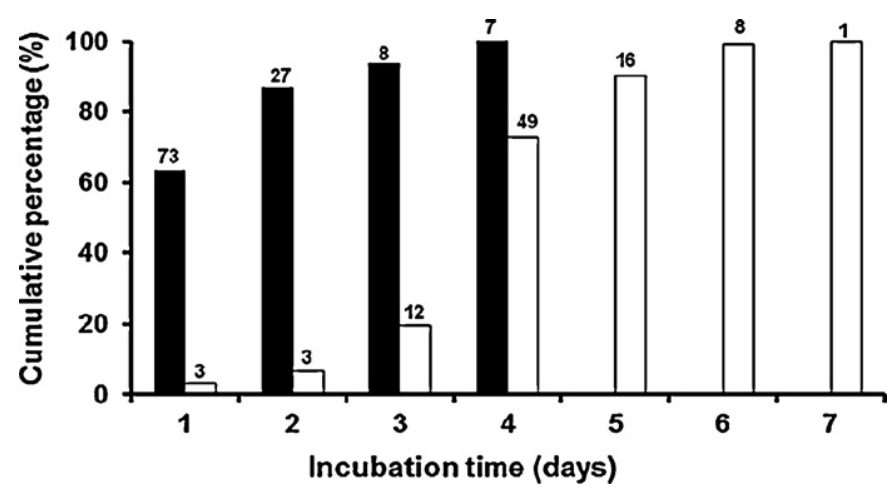

Fig. 2. Influence of agar plate culture incubation time on the identification of furrows left on the agar plates by $S$. stercoralis $(\mathbf{\square})$ or hookworm $(\square)$ larvae. Number of positive cultures per day is presented above the columns. Differences in number of positive APC between species were observed mainly by the first and the fourth day for $S$. stercoralis and hookworm, respectively $(P=0.0001)$. All S. stercoralis and hookworm larvae were confirmed by morphological examination.

\section{Discussion}

A definitive diagnosis of strongyloidiasis is usually made by the detection of larvae in the stool. However, S. stercoralis is one of the most difficult intestinal parasites to diagnose because of the low parasite load and the irregular larvae output in the majority of subclinical infections; thus, the true prevalence of S. stercoralis is underestimated. In the present study, the frequencies of infection were $6.6 \%$ for S. stercoralis and for $7.7 \%$ hookworm. The hookworm infections observed herein may be caused by Necator americanus, since it is the most prevalent species in Bahia, Brazil (Faria, 1972).

The APC method is considered more efficient than other conventional methods adopted in clinical laboratories (Arakaki et al., 1990; De Kaminsky, 1993; Marchi Blatt and Cantos, 2003). In this study, the Baermann-Moraes and spontaneous sedimentation methods failed to detect 11 and 29 cases of strongyloidiasis, respectively, that were detected by agar plate culture. As previously described

Table 2

Characteristics of furrows left on agar plates by larvae of Strongyloides stercoralis and hookworms compared with morphological identification.

\begin{tabular}{|c|c|c|c|c|}
\hline \multirow[t]{2}{*}{ Characteristics of furrows } & \multicolumn{4}{|c|}{ Larvae confirmed by morphology } \\
\hline & S. stercoralis, $n(\%)$ & Hookworm, $n(\%)$ & Mixed, $n(\%)$ & Total \\
\hline Whip-like movement with or without thick furrows ${ }^{\mathrm{a}}$ & $110(95.7)$ & $3(3.3)$ & - & 113 \\
\hline Snake-like gliding $\mathrm{b}$ & $5(4.3)$ & $89(96.7)$ & - & 94 \\
\hline Mixed pattern ${ }^{c}$ & & & $3(100)$ & 3 \\
\hline Total & 115 & 92 & 3 & 210 \\
\hline
\end{tabular}

a Relatively regular sine-curve furrows left by larvae usually in straight line or with a gentle change in direction.

b Relatively irregular twisting furrows with somewhat abrupt changes in direction.

c Both patterns observed on the agar. 
Table 3

Storage time and viability of Strongyloides stercoralis larvae.

\begin{tabular}{|c|c|c|c|c|}
\hline \multirow[t]{2}{*}{ Method } & \multicolumn{4}{|c|}{ Storage time of stool samples at $4^{\circ} \mathrm{C}^{\mathrm{a}}$} \\
\hline & $0 \mathrm{~h}(\%)$ & $24 \mathrm{~h}(\%)$ & $48 \mathrm{~h}(\%)$ & $72 \mathrm{~h}(\%)$ \\
\hline APC & $74(100)$ & $36(48.6)$ & $21(28.4)$ & $17(23)$ \\
\hline Baermann-Moraes & $72(100)$ & $42(58.3)$ & $18(25)$ & $12(16.7)$ \\
\hline
\end{tabular}

a Samples positive for Strongyloides stercoralis and stored at $4{ }^{\circ} \mathrm{C}$ were examined at 4 different time points.

by other authors (Koga et al., 1991; Sato et al., 1995) and confirmed in this study, the APC method is more accurate for identifying $S$. stercoralis larvae in faecal specimens, even when only one sample is examined. However, it is worth noting that 2 samples identified as positive by the Baermann-Moraes were found to be negative by the APC method (data not shown), suggesting the need for the concurrent use of both methods. Moreover, APC was more accurate than the spontaneous sedimentation and Baermann-Moraes methods for the diagnosis of hookworm, as demonstrated in others studies (Dreyer et al., 1996; Knopp et al., 2008). It is important to mention that 8 stool samples with hookworm larvae were identified by Baermann-Moraes. We suppose that samples from patients with reduced bowel movement or constipation could have hookworm rabditiform larvae and/or eggs in advanced-stage development, hatching during the faecal processing in the laboratory.

The time required, higher costs and the technician exposure to filariform larvae, compared to Baermann-Moraes method, are the main reasons that clinical laboratories do not choose APC for the routine diagnosis of strongyloidiasis. However, the high sensitivity of the APC justifies its implementation in clinical laboratories and the exposure to the infective larvae may be reduced by the use of double-walled plates (Arakaki et al., 1990) and by careful manipulation. Moreover, the identification of parasites based both on the different patterns of larvae movement and time required for positivity in APC, may increase sensitivity of the method when larvae are not recovered from cultures, probably due to deep parasite migration on agar. Indeed, in order to optimize the recovery of $S$. stercoralis filariform stage for antigen preparation purposes, in our laboratory, after washing plates, the agar is taken off and submitted to the Baermann-Moraes method, increasing by about $10 \%$ the number of parasites (data not shown).

The rhabditiform and filariform stages of the one parasite species exhibit similar furrow characteristics, but these furrow characteristics differ from those of other species (Arakaki et al., 1990; Jongwutiwes et al., 1999). In the present study, a total of 110 (95.7\%) samples positive for S. stercoralis, 89 (96.7\%) positive for hookworm and 3 (100\%) positive for a mixed infection had concordant results between the furrow patterns and the morphological characteristics of the larvae. The tracks of bacterial growth along the paths of motile larvae of $S$. stercoralis became apparent after one or 2 days of incubation, allowing the characterisation of furrows sooner than for hookworm. By the first day of incubation, 73 (63.5\%) S. stercoralis samples had become positive, in contrast with the 3 (3.3\%) hookworm-positive samples at this time point. This difference in the correlations between $S$. stercoralis and hookworm positivity and time of incubation occurs because hookworm eggs require a few days for hatching and the development of larvae (Jongwutiwes et al., 1999). One explanation for the five (4.3\%) S. stercoralis-positive samples that were not confirmed by furrow characteristics is that these cultures required more than 4 days to form crawling marks on the agar medium, allowing an overgrowth of bacteria colonies and making analysis more difficult. Jongwutiwes et al. (1999) have suggested incubating plates for up to 7 days to increase the detection rate of S. stercoralis. However, in this study, by the fourth day of culture, all samples had already become positive. These variations may be dependent on the parasite load of each individual sample. Moreover, in a few samples, the deep parasite migration on agar may delay the onset of tracks of both species.

Regardless of the availability of immunological tests to support a diagnosis of strongyloidiasis (Machado et al., 2008), definitive diagnosis is based on the identification of larvae in fresh faeces. Complaints concerning access to clinical laboratories, which are usually far from patients' homes or physiological difficulties related to collecting stool samples a few hours before delivery to the laboratory prompt a discussion about accepting refrigerated stools for analysis. In the present study, both the APC and Baermann-Moraes methods lost approximately $50 \%$ of their sensitivity for the detection of S. stercoralis larvae, when samples refrigerated for $24 \mathrm{~h}$ were used. Increasing the storage time at $4{ }^{\circ} \mathrm{C}$ considerably decreased the sensitivity of these methods. Most samples showing positivity even after $72 \mathrm{~h}$ of refrigeration had a high parasite load or, for samples from hyperinfected patients, contained a few filariform infectivestage organisms. The high numbers of rhabditoid larvae along with the presence of very resistant filariform infective stage may be factors that contribute to the maintenance of positivity of refrigerated stools.

In summary, many cases of S. stercoralis infection may be overlooked if only diagnostic conventional methods are used. More accurate parasitological methods, such as ACP, should be recommended for routine laboratory analysis. This was observed herein with the increase of frequency rates for both S. stercoralis and hookworm. Additionally, the failure to detect larvae in a single stool sample does not necessarily indicate the absence of infection; thus, repeated examinations of faeces using sensitive methods is highly recommended, especially in high-risk groups (Siddiqui and Berk, 2001; Steinmann et al., 2007; Hirata et al., 2007; Teixeira et al., 2010). However, asymptomatic chronically infected individuals may not always agree to deliver three or more samples, emphasising the need for high-efficacy diagnostic methods for routine laboratory analysis.

\section{Acknowledgments}

This work was supported by Ministério da Saúde/Fundação de Amparo à Pesquisa do Estado da Bahia (PPSUS/FAPESB) and Conselho Nacional de Desenvolvimento Científico e Tecnológico (CNPq), Brazilian agencies.

\section{References}

Arakaki, T., Iwanaga, M., Kinjo, F., Saito, A., Asato, R., Ikeshiro, T., 1990. Efficacy of agar-plate culture in detection of Strongyloides stercoralis infection. J. Parasitol. $76,425-428$.

Ardiç, N., 2009. An overview of Strongyloides stercoralis and its infections. Mikrobiyol. Bul. 43, 169-177.

Borda, C.E., Rea, M.J., Rosa, J.R., Maidana, C., 1996. Intestinal parasitism in San Cayetano, Corrientes, Argentina. Bull. Pan Am. Health Organ. 30, 227-233.

De Kaminsky, R.G., 1993. Evaluation of three methods for laboratory diagnosis of Strongyloides stercoralis infection. J. Parasitol. 79, 277-280.

Dos Santos Neto, J., 1993. Movement of the rhabditiform larva of Strongyloides stercoralis. Lancet 342, 1310.

Dreyer, G., Fernandes-Silva, E., Alves, S., Rocha, A., Albuquerque, R., Addiss, D., 1996. Patterns of detection of Strongyloides stercoralis in stool specimens: implications for diagnosis and clinical trials. J. Clin. Microbiol. 34, 2569-2571.

Faria, J.A.S., 1972. Prevalência de helmintos em escolares de 7-14 anos na cidade do Salvador. Rev. Soc. Bras. Med. Trop. 6, 261-264. 
Gamboa, M.I., Kozubsky, L.E., Costas, M.E., Garraza, M., Cardozo, M.I., Susevich, M.L., Magistrello, P.N., Navone, G.T., 2009. Associations between geohelminths and socioenvironmental conditions among different human populations in Argentina. Rev. Panam. Salud Publica 26, 1-8.

Hirata, T., Nakamura, H., Kinjo, N., Hokama, A., Kinjo, F., Yamane, N., Fujita, J., 2007. Increased detection rate of Strongyloides stercoralis by repeated stool examinations using the agar plate culture method. Am. J. Trop. Med. Hyg. 77, 683-684.

Jongwutiwes, S., Charoenkorn, M., Sitthichareonchai, P., Akaraborvorn, P., Putaporntip, C., 1999. Increased sensitivity of routine laboratory detection of Strongyloides stercoralis and hookworm by agar-plate culture. Trans. R. Soc. Trop. Med. Hyg. 93, 398-400.

Knopp, S., Mgeni, A.F., Khamis, I.S., Steinmann, P., Stothard, J., Rollinson, D., Marti, H., Utzinger, J., 2008. Diagnosis of soil-transmitted helminths in the era of preventive chemotherapy: effect of multiple stool sampling and use of different diagnostic techniques. PLoS Negl. Trop. Dis. 2, e331.

Kobayashi, J., Hasegawa, H., Soares, E., Toma, H., Dacal, A., Brito, M., Yamanaka, A., Foli, A., Sato, Y., 1996. Studies on prevalence of Strongyloides infection in Holambra and Maceió, Brazil, by the agar plate faecal culture method. Rev. Inst. Med. Trop. São Paulo 38, 279-284.

Koga, K., Kasuya, S., Khamboonruang, C., Sukavat, K., Nakamura, Y., Tani, S., Ieda, M., Tomita, K., Tomita, S., Hattan, N., 1990. An evaluation of the agar plate method for the detection of Strongyloides stercoralis in northern Thailand. J. Trop. Med. Hyg. 93, 183-188.

Koga, K., Kasuya, S., Khamboonruang, C., Sukhavat, K., Ieda, M., Takatsuka, N., Kita, K., Ohtomo, H., 1991. A modified agar plate method for detection of Strongyloides stercoralis. Am. J. Trop. Med. Hyg. 45, 518-521.

Koga, K., Kasuya, S., Ohtomo, H., 1992. How effective is the agar plate method for Strongyloides stercoralis? J. Parasitol. 78, 155-156.

Kramme, S., Nissen, N., Soblik, H., Erttmann, K., Tannich, E., Fleischer, B., Panning, M., Brattig, N., 2011. Novel real-time PCR for the universal detection of Strongyloides species. J. Med. Microbiol. 60, 454-458.
Machado, E.R., Teixeira, E.M., Gonçalves-Pires, M.d.F., Loureiro, Z.M., Araújo, R.A Costa-Cruz, J.M., 2008. Parasitological and immunological diagnosis of Strongyloides stercoralis in patients with gastrointestinal cancer. Scand. J. Infect. Dis. 40, $154-158$.

Marchi Blatt, J., Cantos, G., 2003. Evaluation of techniques for the diagnosis of Strongyloides stercoralis in human immunodeficiency virus (HIV) positive and HIV negative individuals in the city of Itajaí, Brazil. Braz. J. Infect. Dis. 7, 402-408.

Menezes, A., Lima, V., Freitas, M., Rocha, M., Silva, E., Dolabella, S., 2008. Prevalence of intestinal parasites in children from public daycare centers in the city of Belo Horizonte, Minas Gerais, Brazil. Rev. Inst. Med. Trop. São Paulo 50, 57-59.

Olsen, A., Van Lieshout, L., Marti, H., Polderman, T., Polman, K., Steinmann, P., Stothard, R., Thybo, S., Verweij, J., Magnussen, P., 2009. Strongyloidiasis-the most neglected of the neglected tropical diseases? Trans. R. Soc. Trop. Med. Hyg. 103, 967-972.

Salazar, S., Gutierrez, C., Berk, S., 1995. Value of the agar plate method for the diagnosis of intestinal strongyloidiasis. Diagn. Microbiol. Infect. Dis. 23, 141-145.

Sato, Y., Kobayashi, J., Toma, H., Shiroma, Y., 1995. Efficacy of stool examination for detection of Strongyloides infection. Am. J. Trop. Med. Hyg. 53, 248-250.

Siddiqui, A., Berk, S., 2001. Diagnosis of Strongyloides stercoralis infection. Clin. Infect. Dis. 33, 1040-1047.

Steinmann, P., Zhou, X.-N., Du, Z.-W., Jiang, J.-Y., Wang, L.-B., Wang, X.-Z., Li, L.-H. Marti, H., Utzinger, J., 2007. Occurrence of Strongyloides stercoralis in Yunnan Province, China, and Comparison of Diagnostic Methods. PLoS Negl. Trop. Dis. 1, e75.

Teixeira, M.C., Inês, E.J., Pacheco, F.T., Silva, R.K., Mendes, A.V., Adorno, E.V., Lima, F.M., Soares, N.M., 2010. Asymptomatic Strongyloides stercoralis hyperinfection in an alcoholic patient with intense anemia. J. Parasitol. 96, 833-835.

Uparanukraw, P., Phongsri, S., Morakote, N., 1999. Fluctuations of larval excretion in Strongyloides stercoralis infection. Am. J. Trop. Med. Hyg. 60, 967-973.

Verweij, J.J., Canales, M., Polman, K., Ziem, J., Brienen, E.A., Polderman, A.M., Van Lieshout, L., 2009. Molecular diagnosis of Strongyloides stercoralis in faecal samples using real-time PCR. Trans. R. Soc. Trop. Med. Hyg. 103, 342-346. 